\title{
Essential role of Gas6 for glomerular injury in nephrotoxic nephritis
}

\author{
Motoko Yanagita, ${ }^{1}$ Yoshikazu Ishimoto, ${ }^{2}$ Hidenori Arai, ${ }^{1}$ Kojiro Nagai, ${ }^{1}$ Tsuyoshi Ito, ${ }^{2}$ \\ Toru Nakano, ${ }^{2}$ David J. Salant,${ }^{3}$ Atsushi Fukatsu, ${ }^{4}$ Toshio Doi, ${ }^{5}$ and Toru Kita ${ }^{1}$
}

${ }^{1}$ Department of Geriatric Medicine, Kyoto University, Kyoto, Japan
2 Discovery Research Laboratory, Shionogi Co., Osaka, Japan
${ }^{3}$ Renal Section, Boston University Medical Center, Boston, Massachusetts, USA
${ }^{4}$ Department of Artificial Kidneys, Kyoto University, Kyoto, Japan
${ }^{5}$ Department of Laboratory Medicine, Tokushima University, Tokushima, Japan

Growth-arrest specific gene 6 (Gas6) is a vitamin $\mathrm{K}$-dependent growth factor for mesangial and epithelial cells. To investigate whether Gas 6 is essential for progressive glomerular injury, we constructed $\mathrm{Gas}^{-/-}$mice and examined the role of Gas6 in accelerated nephrotoxic nephritis (NTN), a model of progressive glomerulonephritis. We found less mortality and proteinuria in $\mathrm{Gas}^{-/-}$mice than in wild-type mice following injection of nephrotoxic serum. Glomerular cell proliferation, glomerular sclerosis, crescent formation, and deposition of fibrin/fibrinogen in glomeruli were also reduced in $\mathrm{Gas}^{-/-}$mice. Furthermore, administering $\mathrm{Gas}^{-/-}$mice recombinant wild-type Gas6, but not Gas6 lacking a previously characterized $\mathrm{N}$-terminal $\gamma$-carboxyl group, induced massive proteinuria, glomerular cell proliferation, and glomerulosclerosis, comparable to responses seen in wildtype mice. These data indicate that Gas6 induces glomerular cell proliferation in NTN and suggest that this factor contributes to glomerular injury and the progression of chronic nephritis.

J. Clin. Invest. 110:239-246 (2002). doi:10.1172/JCI200214861.

\section{Introduction}

Glomerular cell proliferation accompanies a wide variety of renal diseases and is usually associated with matrix expansion that leads to the development of end-stage kidney disease (1-3). Therefore, extensive efforts have been made to elucidate growth factors and cytokines involved in glomerular cell proliferation. Among potential mitogens for glomerular cells we have focused on Gas6, a vitamin K-dependent growth factor whose action is inhibited by the anticoagulant warfarin (4-6). The activities of Gas 6 depend on $\gamma$-carboxylation of glutamate residues at its $\mathrm{N}$ terminus $(5,6)$. Recently we showed that Gas6 is an autocrine growth factor for mesangial cells, and that warfarin and the extracellular domain of Axl (a receptor for Gas6) inhibit mesangial cell proliferation by specific blockade of the Gas6-mediated pathway in a mesangial-proliferative model of glomerulonephritis

Received for publication December 14, 2001, and accepted in revised form June 4, 2002.

Address correspondence to: $\mathrm{H}$. Arai, Department of Geriatric Medicine, Kyoto University School of Medicine,

54 Kawahara-cho, Shogoin, Sakyo-ku, Kyoto 606-8507, Japan. Phone: 81-75-751-3463; Fax: 81-75-751-3463;

E-mail: harai@kuhp.kyoto-u.ac.jp.

Motoko Yanagita and Yoshikazu Ishimoto contributed equally to this work.

Conflict of interest: No conflict of interest has been declared. Nonstandard abbreviations used: Glomerulonephritis (GN); nephrotoxic nephritis (NTN); nephrotoxic serum (NTS); phosphoglycerate kinase-1 (Pgk-1); glomerular basement membrane(GBM); periodic acid-Schiff (PAS); proliferating cell nuclear antigen (PCNA); recombinant Gas6 (rGas6); Gas6 lacking $\gamma$-carboxylation (Gla $\Delta$ Gas6).
(GN), Thy1 GN $(7,8)$. Moreover, administration of warfarin and the extracellular domain of Axl abolish the induction of PDGF-B in Thy1 GN. Thus, Gas6 seems to be not only a mitogen for mesangial cells, but also one that plays a critical role in the progression of glomerular diseases by modulating the expression of other growth factors. Initially, linear deposition of injected antibodies on glomerular basement membranes (GBM), rapid elevation of blood urea nitrogen, infiltration of lymphocytes and monocytes, and glomerular hypercellularity are observed, while production and deposition of antibodies against the injected heterologous IgG, glomerulosclerosis, and crescent formation are observed in the later phase of nephrotoxic nephritis (NTN) (9).

Although our findings in the Thy $1 \mathrm{GN}$ model suggest that Gas6 may be a new and specific target for therapeutic intervention in various kidney diseases, the Thy 1 GN model is self-limited and spontaneously reversible. Therefore, it might be anticipated that the benefits of neutralizing Gas6 would not be found in progressive forms of GN. Because most serious glomerular diseases are progressive and lead to chronic renal failure, we set out to explore the possibility that Gas6 may be involved in a progressive type of proliferative $\mathrm{GN}$ that is associated with prolonged proteinuria and glomerular damage. For that purpose we used a well-established model of crescentic GN, accelerated NTN in the mouse (9). NTN is a progressive form of GN in which inflammatory cell infiltration and proliferation of intrinsic glomerular cells contribute to glomerular hypercellularity, the formation of crescentic lesions in the urinary space, and glomerular sclerosis. It is induced by injecting preimmunized 
mice with heterologous nephrotoxic serum (NTS), which has reactivity to several glomerular cell and basement membrane antigens (10).

In the studies reported here, we created $\mathrm{Gas}^{-/-}$mice and used them to examine the role of Gas 6 in murine NTN. Our findings demonstrate that Gas 6 is essential for the full expression of progressive glomerular injury in this model.

\section{Methods}

Construction of targeting vector. The mouse Gas6 gene was cloned from a 129/SvJ genomic library (Stratagene, La Jolla, California, USA) using a 0.2-kb cDNA fragment encompassing the initial ATG codon of mouse Gas 6 as a probe (6). The 3.0-kb EcoRI-EcoRI and $3.5-\mathrm{kb}$ BamHI-BamHI genomic fragments derived from the isolated clone were used for the construction of the targeting vector, along with a neomycin-resistance gene driven by the phosphoglycerate kinase-1 (Pgk-1) promoter (Pgk-neor) and a diphtheria toxin A-fragment gene driven by the $\mathrm{MC} 1$ promoter as positive and negative selection markers, respectively (Figure 1) (11). Using this construct, homologous recombination results in the replacement of the EcoRI-BamHI genomic fragment that includes the translation starting codon in the Pgk-neo ${ }^{r}$ cassette, resulting in abolition of Gas6 expression.

Generation of Gas6 knockout mice. The embryonic stem cell line used in this study was E14 derived from 129/Ola mice. The targeting experiment and generation of mutant mice were performed as described previously (12). The germline chimera was backcrossed for 17 generations with $\mathrm{C} 57 \mathrm{BL} / 6$ mice to obtain $\mathrm{Gas}^{+/-}$mice with a C57BL/ 6 background. The resulting $\mathrm{Gas}^{+/-} \mathrm{F}_{17}$ mice were then intercrossed to generate the homozygous $\mathrm{Gas}^{-/-}$mice. Additional control inbred C57BL/6 mice were obtained from Shimizu Laboratory Animal Center (Hamamatsu, Japan). All mice were housed under specific pathogen-free conditions. All animal experiments were performed in accordance with institutional guidelines, and the Review Board of Kyoto University granted ethical permission to perform this study.

Southern blotting. The genotypes of mice were determined by Southern blot analysis of DNA prepared from tails. Genomic DNAs were digested overnight with EcoRV and electrophoresed with $0.8 \%$ agarose gels. The DNAs were transferred to nylon membranes and probed with a 0.8-kb BamHI-HindIII fragment labeled with $\left[\alpha-{ }^{32} \mathrm{P}\right] \mathrm{dCTP}$. Membranes were then analyzed using a Fujix BAS2000 Bio-Image Analyzer (Fuji Photo Film Co. Ltd., Tokyo, Japan).

Preparation of NTS. Sheep were immunized with glomerular lysates prepared from Sprague-Dawley rat kidneys, as described (9). NTS was heat-inactivated at $56^{\circ} \mathrm{C}$ for 45 minutes and then absorbed overnight with mouse red blood cells. Before use, the preparation was sterilized by passage through a $0.2-\mu \mathrm{M}$ filter.

Induction of accelerated NTN. Male wild-type or Gas $6^{-/-}$ mice ( 8 weeks old) weighing $20-25$ g were sensitized by subcutaneous injection of $1 \mathrm{mg}$ normal sheep $\operatorname{IgG}$ in Freund's complete adjuvant in divided doses into each flank. Five days later, mice were injected with $0.1 \mathrm{ml}$ of NTS daily for 3 days. At intervals from 3 to 21 days after the first dose of NTS, groups of mice (six to eight per group) were sacrificed, blood was collected, and the kidneys were removed for the experiments.

Northern blotting of Gas6. Whole-kidney RNA was isolated using RNeasy (QIAGEN Inc., Valencia, California, USA). Twenty micrograms of denatured RNA was electrophoresed through formaldehyde $1 \%$ agarose gel and transferred to nylon membranes as previously described (10). Membranes were hybridized with the EcoRI-BglII 0.7-kb fragment of mouse Gas $6 \mathrm{cDNA}$ radiolabeled with [32 P]dCTP $(10 \mathrm{mCi} / \mathrm{ml}$; Amersham Pharmacia Biotech, Buckinghamshire, United Kingdom) by random primer extension. All Northern blots were repeated at least three times with RNA from different sets of animals.

Western blotting analysis of Gas6, STAT3, and phosphoSTAT3. Whole-kidney protein was homogenized in RIPA buffer $(50 \mathrm{mM}$ Tris at $\mathrm{pH} 7.5,150 \mathrm{mM} \mathrm{NaCl}, 1 \%$ Nonidet P40, 0.25\% SDS, $1 \mathrm{mM} \mathrm{Na}_{3} \mathrm{VO}_{4}, 2 \mathrm{mM}$ EDTA, $1 \mathrm{mM}$ PMSF, and $10 \mu \mathrm{g} / \mathrm{ml}$ aprotinin) and rotated for 1 hour at $4^{\circ} \mathrm{C}$. After centrifugation of the samples, the supernatants were used as total cell lysates. Sixty micrograms of each sample was applied to SDS-PAGE gels and immunoblotted as described (8). Rabbit polyclonal antibody against rat Gas6, which cross-reacts with mouse Gas6, was made as described (5). Rabbit anti-STAT3 and anti-phospho-STAT3 antibodies were from Cell Signaling Technology Inc. (Beverly, Massachusetts, USA).

Concentrations of albumin in serum and urine. Urinary albumin excretion was measured at intervals from 0 to 21 days in 24-hour urine collection samples from mice housed in individual metabolic cages. During the urine collection, mice were allowed free access to food and water. Albumin concentration in the urine was assayed using the Albuwell kit (Exocell Inc., Philadelphia, Pennsylvania, USA). Serum concentration of albumin was analyzed using Albumin HR-II kit (Wako Pure Chemical Industries Ltd., Osaka, Japan).

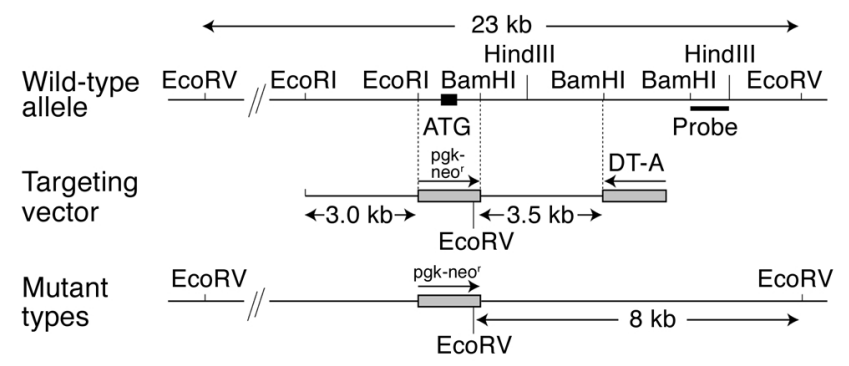

\section{Figure 1}

Genomic structure of the mouse Gas6 gene and the targeting vector. The 3.0-kb EcoRI-EcoRI and 3.5-kb BamHI-BamHI genomic fragments were used for the construction of the targeting vector. Homologous recombination results in the replacement of the EcoRI-BamHI genomic fragment including the translation starting codon in the Pgk-neor cassette, resulting in loss of Gas 6 expression. DT-A, diptheria toxin A. 


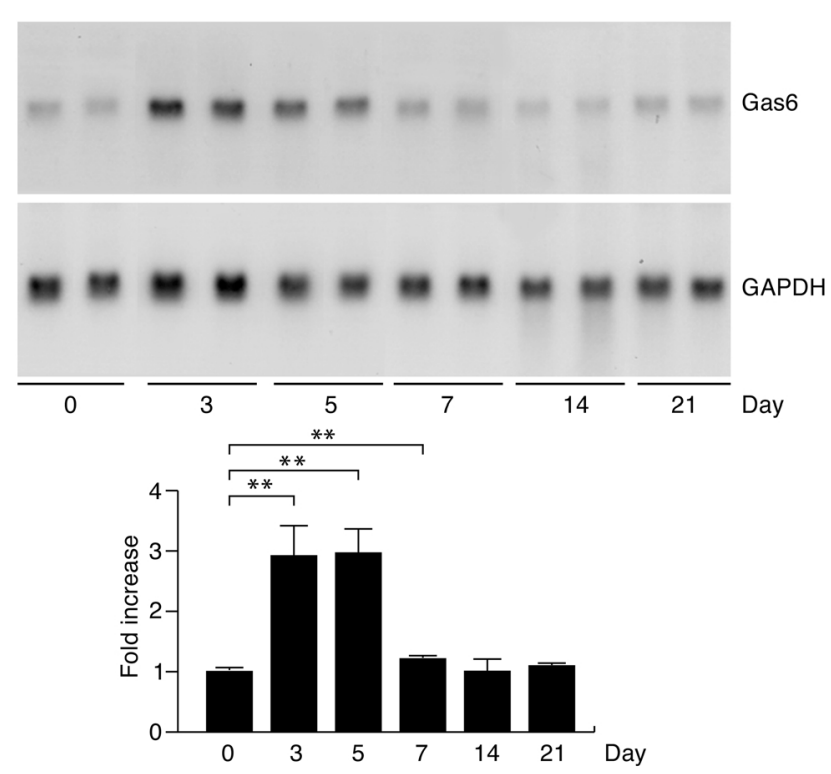

Figure 2

Expression of Gas 6 in the proliferative phase of NTN. RNA collected from four to eight representative mice on days $0-21$ were subjected to Northern blotting. The expression of GAPDH served as a control for RNA loading. Representative results are shown in the upper panels. The graph shows densitometric analysis of Gas 6 mRNA expression after normalization by the expression of GAPDH. The experiments were repeated three times and representative data are shown. ${ }^{*} P<0.01$.

Histologic studies. Kidney halves were fixed in methyl Carnoy's solution and embedded in paraffin. Sections $(2 \mu \mathrm{m})$ were stained with periodic acid-Schiff(PAS) and periodic acid-methenamine silver for routine histology. The remaining kidney half was snap-frozen and used for immunohistochemical study. All morphologic evaluations were performed in a blinded fashion using six to eight kidneys per group for each time point. We evaluated at least 40 glomeruli per kidney for sclerosis and crescent formation by light microscopy. Percentages of glomeruli with crescents and those with more than 50\% sclerotic area positive for PAS and periodic acid-methenamine silver were calculated The expression of proliferating cell nuclear antigen (PCNA), phospho-STAT3, and fibrin/fibrinogen was evaluated by immunostaining. Localization of fibrin/fibrinogen was semiquantitated and given a score of $0-3$ as follows: 0 , no staining; 1 , up to one-third; 2 , one-third to twothirds; and 3, more than two-thirds of glomerular cross section positive for fibrin/fibrinogen. FITC-labeled rabbit anti-sheep antibody and FITC-labeled rabbit anti-human fibrinogen (known to cross-react with mouse fibrin and fibrinogen) were purchased from DAKO Corp. (Carpinteria, California, USA). Rabbit anti-PCNA antibody and rabbit anti-phospho-STAT3 antibody were from Santa Cruz Biotechnology Inc. (Santa Cruz, California, USA) and New England Biolabs Inc. (Beverly, Massachusetts, USA), respectively.

Estimation of circulating anti-sheep IgG antibody. The levels of circulating anti-sheep IgG were estimated by ELISA. ELISA plates (Nippon InterMed KK, Tokyo,
Japan) coated with sheep IgG (Sigma-Aldrich, St. Louis, Missouri, USA) were incubated with test plasma that was diluted to 1:1,000. After being washed extensively with PBS containing $0.05 \%$ Tween 20 , the plates were incubated with horseradish peroxidase-conjugated rat anti-mouse IgG (Jackson ImmunoResearch Laboratories, West Grove, Pennsylvania, USA) diluted to 1:5,000. A kinetic analysis of absorbance at $650 \mathrm{~nm}$ was performed using 3,3',5,5'-tetramethylbenzidine (Nacalai Tesque Inc., Kyoto, Japan) as a substrate. The level of anti-rabbit IgG was estimated by comparing the initial velocity of the increase in absorbance at $650 \mathrm{~nm}$.

Injection of Gas 6 to $\mathrm{Gas}^{-/-}$mice. Recombinant Gas6 was purified as described previously $(5,6)$. Two micrograms of Gas 6 was injected daily into Gas $6^{-/-}$mice from day 4 to the day of sacrifice. As a negative control, the same amount of inactive Gas 6 whose Gla domain was not $\gamma$-carboxylated (Gla $\Delta$ Gas6) was injected.

Statistical analyses. Statistical significance was determined using the Student $t$ test. $P<0.05$ was considered significant. Data are expressed as mean \pm SD. Analysis was performed by simple regression using the StatView program (Abacus Concepts Inc., Berkeley, California, USA).

\section{Results}

Expression of Gas6 during the proliferative phase of NTN. First we examined expression of Gas6 in the kidney during the course of NTN in wild-type mice. As we found in the Thy1 model of acute GN in rats (10), the expression of Gas 6 mRNA was increased by about threefold on day 3 and day 5 in the kidneys of mice with NTN (Figure 2). In this phase of NTN, the glomeruli become significantly hypercellular because of cell migration and cell proliferation (13).

Generation and characterization of $\mathrm{Gas}^{-/-}$mice. To investigate the role of Gas6 in NTN, we generated Gas6 knockout mice (Gas6 $6^{-1-}$ mice) by deleting the

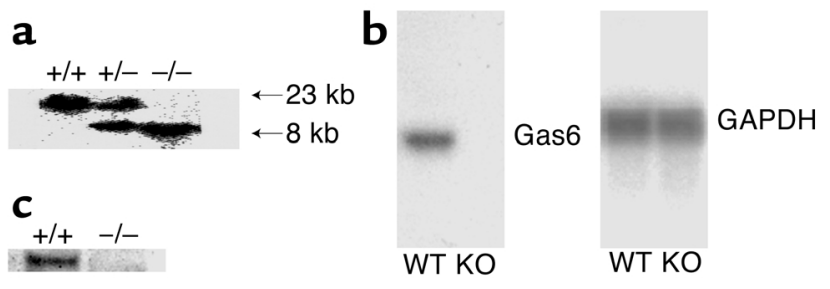

\section{Figure 3}

Lack of Gas 6 mRNA and protein in Gas $6^{-/-}$mice. (a) Southern blotting of genomic DNA from the progeny of intercrosses of Gas 6 heterozygous mutant mice. The genomic DNA from wild-type mice $(+/+)$, heterozygous mice $(+/-)$, and Gas $6^{-/-}$mice $(-/-)$was digested with EcoRV and hybridized with the probe described in Figure 1. The upper and lower bands correspond to the wild-type and targeted alleles, respectively. (b) Northern blotting of Gas 6 mRNA in wild-type (WT) and $\mathrm{Gas}^{-/-}(\mathrm{KO})$ mice. mRNA prepared from kidney of wildtype and $\mathrm{Gas}^{-/-}$mice was subjected to Northern blotting. GAPDH was used as internal control. (c) Western blotting of Gas 6 in wildtype and $\mathrm{Gas}^{-/-}$mice, using lysates prepared from spleen. 

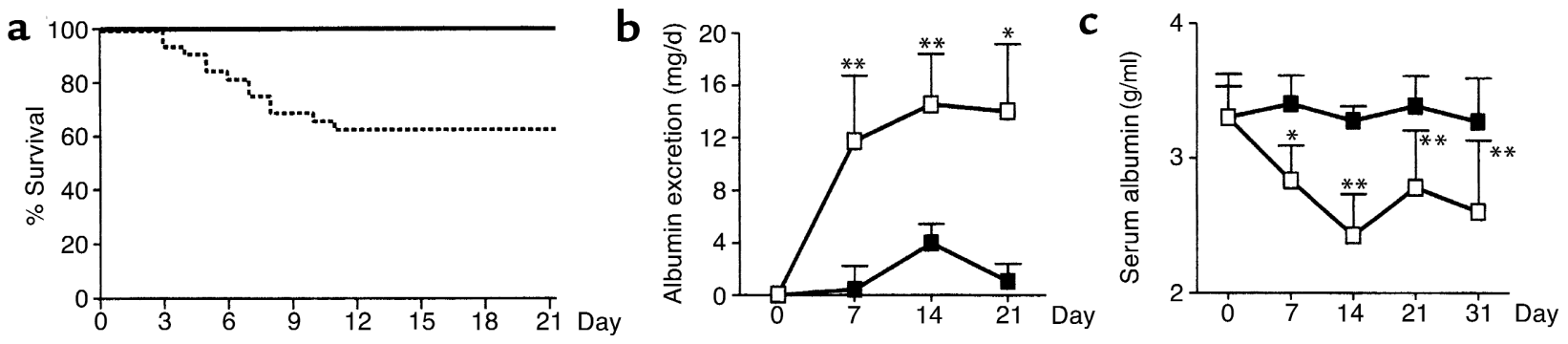

\section{Figure 4}

Gas6 ${ }^{-/-}$mice showed less renal injury than did wild-type mice. (a) Survival curves of wild-type (broken line) or Gas6 $6^{-/-}$(solid line) mice after injection of NTS. Each group consisted of 16 mice. (b) Urinary albumin levels of wild-type (open squares) and Gas $6^{-/-}$(filled squares) mice after injection of anti-GBM Ab on the day indicated. Each group consisted of five mice on day 0 , nine mice on day 7 , eight mice on day 14, and six mice on day 21. Data are expressed as mean \pm SD. (c) Serum albumin levels in wild-type (open squares) and Gas6 $6^{-/-}$(filled squares) mice on the day indicated during the course of NTN. Each group consisted of four mice with the exception of a group of six mice on day 21. Data are expressed as mean \pm SD. ${ }^{*} P<0.05$. ${ }^{*} P<0.01$.

transcription initiation codon, the signal peptide, and the Gla domain that is essential for the biological function of Gas6 (Figure 1). We confirmed correct targeting at the DNA, RNA, and protein levels (Figure 3). Phenotype of $\mathrm{Gas}^{-/-}$mice. Homozygous Gas $6^{-/-}$mice were born at the expected Mendelian frequency. The Gas $6^{-1-}$ mice were viable, fertile, and appeared healthy. There was no difference in blood cell composition or plasma lipid composition between wild-type and mutant mice (data not shown). Necropsy and microscopic examination of major tissues revealed no significant pathology in $\mathrm{Gas}^{-/-}$mice.

Gas $6^{-1-}$ mice with NTN showed less mortality, less albuminuria, and higher serum albumin. To determine the effects of Gas 6 deficiency on the development and progression of chronic renal disease, NTN was induced in both Gas $6^{-/-}$and wild-type mice. In this model, $37.5 \%$ of wildtype mice died between day 3 and day 11, while all $\mathrm{Gas}^{-/-}$mice survived until day 21 (Figure 4a). Bloody urine was found in the bladders of wild-type mice that died. In wild-type mice with NTN, excretion of urinary albumin was increased on day 7 and reached a plateau on day 14. In contrast, much less proteinuria was observed in $\mathrm{Gas}^{-/-}$mice at each timepoint (Figure 4b). In wild-type mice, severe proteinuria was accompanied by a reduction in the serum albumin level, whereas in $\mathrm{Gas}^{-/}$mice, the serum albumin level remained unchanged (Figure 4c).

Gas6 $6^{-1-}$ mice with NTN showed less glomerular injury. We found extensive glomerulosclerosis and crescent formation 21 days after the induction of NTN in wild-type mice (Figure 5a). We also noticed more thrombus formation in the wild-type mice. Consistent with the urinary protein data, glomerulosclerosis and crescent formation were markedly reduced in $\mathrm{Gas}^{-/-}$mice. Semiquantitative analysis of renal tissue damage revealed that glomerular injury was ameliorated in the $\mathrm{Gas}^{-/-}$mice (Figure $5 \mathrm{~b}$ ). Gas $6^{-/}$mice developed fewer crescents (30\% of wild-type mice vs. $11 \%$ of $\mathrm{Gas}^{-/-}$mice, $\left.P<0.01\right)$ and less glomerulosclerosis ( $37 \%$ of wild-type mice vs. $13 \%$ of Gas $6^{-/-}$mice, $P<0.05)$ than did wild-type mice.

Gas6 $6^{-1}$ mice with NTN showed less proliferative response. Because Gas6 is a mitogen for epithelial and mesangial cells $(7,14,15)$ and is considered to induce the proliferative response, we examined cell proliferation in $\mathrm{Gas}^{-/-}$ mice and wild-type mice during the early phase of NTN.
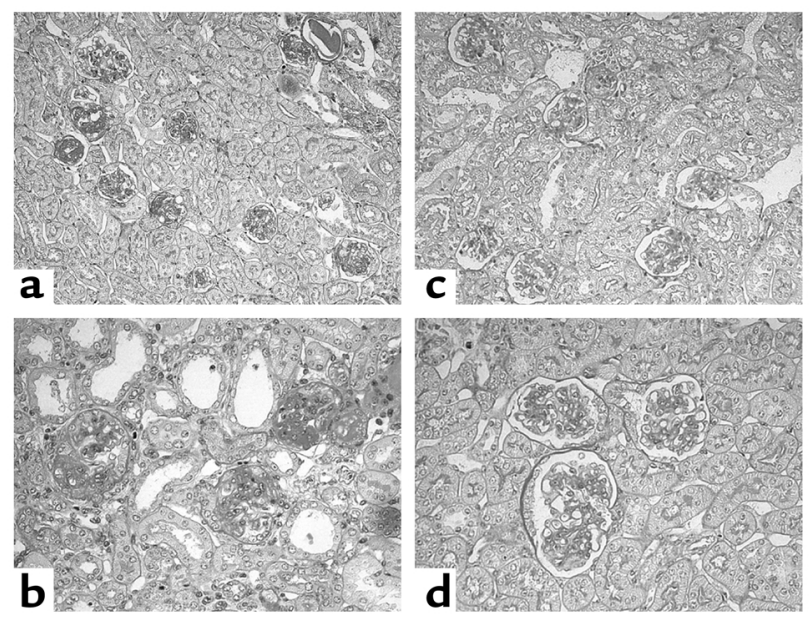

e

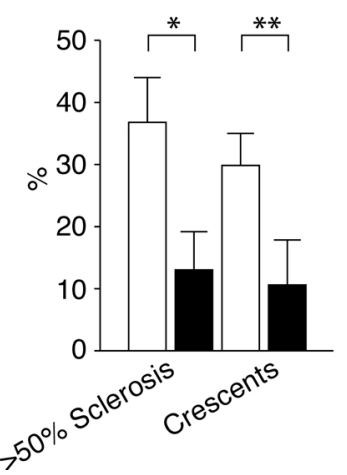

\section{Figure 5}

Representative renal histology and quantitation of glomerulosclerosis and crescent formation. (a-d) Renal histology on day 21 (PAS staining). $\mathbf{a}$ and $\mathbf{b}$, wildtype mice. $\mathbf{c}$ and $\mathbf{d}$, Gas $6^{-1-}$ mice. Magnification: top panels, $\times 100$; bottom panels, $\times 400$. (e) Quantitative assessment of glomerulosclerosis and crescent formation in wild-type and Gas6 $6^{-/-}$mice. Solid bars represent Gas $6^{-/-}$mice, while open bars represent wild-type mice. Each group contained eight mice, and 40 glomeruli per mouse were evaluated in a blinded fashion. ${ }^{*} P<0.05$. ${ }^{*} P<0.01$. 


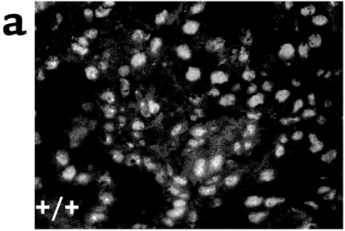

b
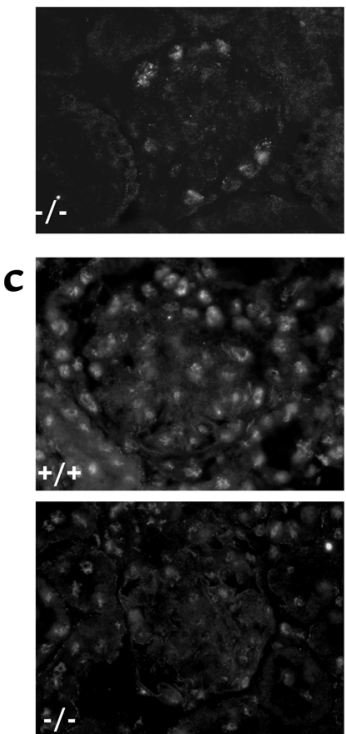

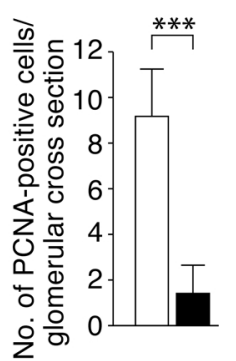

d

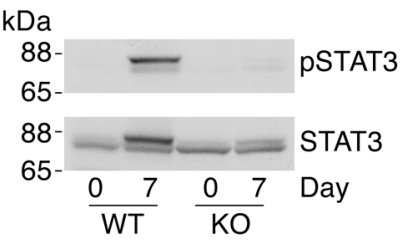

Immunostaining for PCNA on day 7 showed intensive nuclear staining in the kidneys of wild-type mice, whereas scarce staining was observed in $\mathrm{Gas}^{-/-}$mice (Figure 6a). Numbers of PCNA-positive cells per glomerular cross section were significantly smaller in $\mathrm{Gas}^{-/-}$mice than in wild-type mice (9.2 in wild-type mice vs. 1.4 in Gas $6^{-/}$mice, $P<0.05$ ) (Figure 6b). Consistent with the results of PCNA staining, immunostaining for phosphorylated STAT3, which is one of the downstream targets of Gas6 (16), revealed intense nuclear staining in wild-type mice, whereas it was hardly found in $\mathrm{Gas}^{-/-}$ mice (Figure 6c). The difference in STAT3 phosphorylation was also confirmed by immunoblotting. The upper panel of Figure $6 \mathrm{~d}$ shows that STAT3 is heavily phosphorylated in the kidney lysates of wild-type mice on day 7 , whereas its phosphorylation is hardly detectable in the lysates of $\mathrm{Gas}^{-/-}$mice on the same day. The lower panel of Figure $6 \mathrm{~d}$ shows that the amount of STAT3 protein was increased on day 7 in wild-type mice, but not in

\section{Figure 7}

Immunofluorescence staining of fibrin/fibrinogen and sheep IgG of wildtype and Gas6 $6^{-/-}$mice. (a) Immunohistological staining for fibrin/fibrinogen in wild-type and $\mathrm{Gas}^{-/-}$mice sacrificed on day 21. Magnification, $\times 200$. (b) Quantitative assessment of fibrin and fibrinogen staining of wild-type and Gas6 $6^{-1}$ mice. Each group contained eight mice, and 40 glomeruli per mouse were evaluated in a blinded fashion. Data are expressed as mean $\pm \mathrm{SD}$. ${ }^{*}{ }^{*} P<0.001$. (c) Representative immunostaining for sheep $\lg G$ in mice sacrificed on day 7 . Magnification, $\times 40$. Filled bars represent $\mathrm{Gas}^{-/-}$mice, open bars represent wild-type mice. (d) Circulating titers of mouse anti-sheep IgG 21 days after NTS injection were measured by ELISA in both wild-type and $\mathrm{Gas}^{-/-}$mice. Serum dilutions were 1:1,000. Each group consisted of six mice, and data are expressed as mean \pm SD. NS, not significant.

Figure 6

Proliferative response in wild-type and $\mathrm{Gas}^{-1-}$ mice with NTN. (a) Immunostaining for PCNA in wild-type and Gas $6^{-1-}$ mice sacrificed on day 7. Magnification, $\times 200$. (b) Number of PCNA-positive cells per glomerulus in wild-type and Gas6 $6^{-/-}$mice. Solid bars represent Gas6 $6^{-/-}$ mice, while open bars represent wild-type mice. Each group contained eight mice, and 40 glomeruli per mouse were evaluated in a blinded fashion. Data are expressed as mean \pm SD. ${ }^{*} * P<0.001$. (c) Immunostaining for phospho-STAT3 in wild-type and $\mathrm{Gas}^{-/-}$mice sacrificed on day 7. Magnification, $\times 200$. (d) Immunoblotting of kidney protein from wildtype and $\mathrm{Gas}^{-/-}$mice with anti-STAT3 and anti-phospho-STAT3 antibodies. Mice were sacrificed on the day indicated, and kidney proteins were subjected to immunoblotting with anti-STAT3 and anti-phosphoSTAT3 (pSTAT3) antibodies. Representative immunoblotting is shown from four independent experiments.

Gas6 $6^{-/-}$mice. The upper bands in the lower panel correspond to phosphorylated STAT3. We also measured glomerular cell number on days 0 (approximately 18 cells/glomerulus), 7, 14, and 21, and found more cells in wild-type mice than in $\mathrm{Gas}^{-/-}$mice, although statistical significance was attained only on day $14(30.3 \pm 2.0$ in wild-type vs. $25.1 \pm 0.6$ in $\mathrm{Gas}^{-/-}$mice, $P<0.05$ ).

Gas $6^{-/-}$mice with NTN showed less fibrin/fibrinogen deposition. Immunostaining for fibrin/fibrinogen revealed much less glomerular fibrin deposition in $\mathrm{Gas}^{-/-}$mice than was seen in wild-type mice (Figure 7a). Semiquantitative analysis of fibrin/fibrinogen staining showed a significant reduction of deposition in $\mathrm{Gas}^{-{ }^{--}}$ mice compared with wild-type mice. (Figure 7b).

a
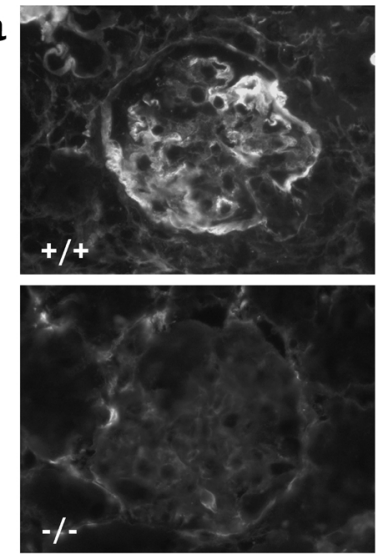

C
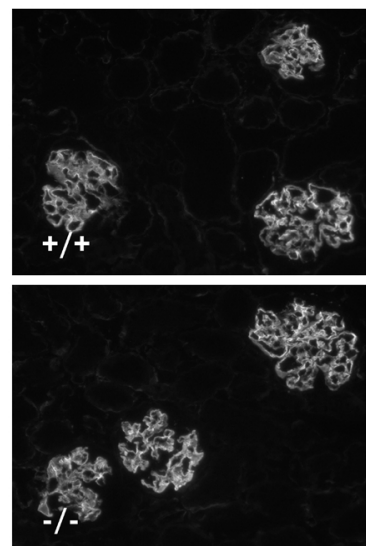

b

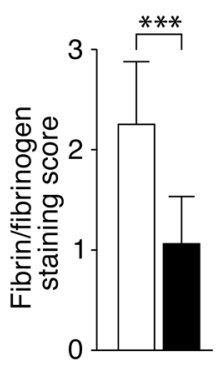

d

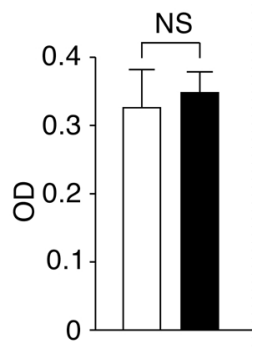


a
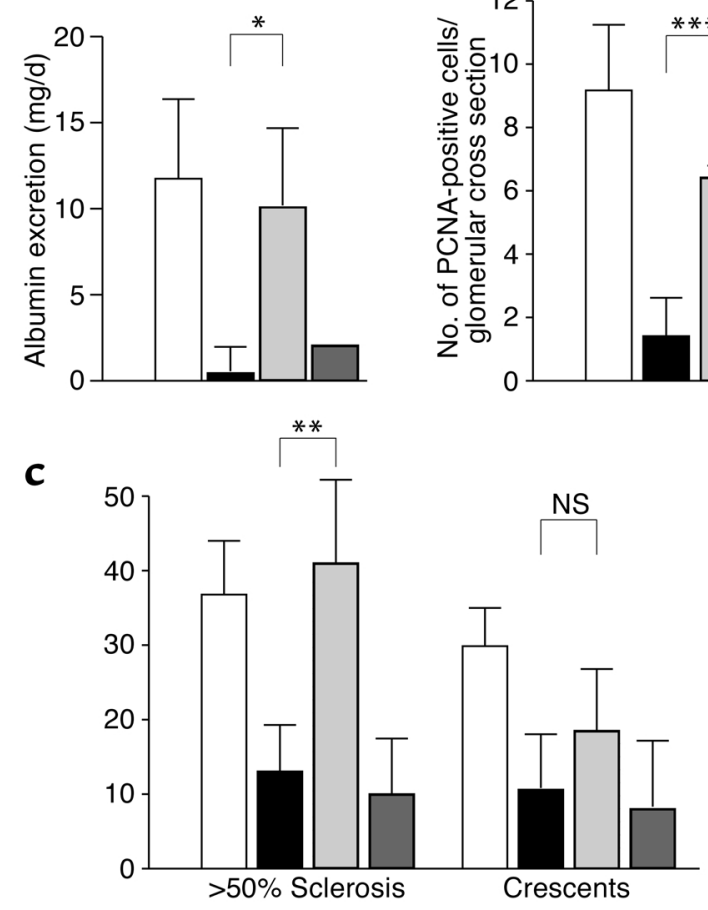

\section{Figure 8}

Induction of glomerular injury by rGas 6 but not by Gla $\Delta$ Gas 6 in Gas $6^{-/-}$mice. (a) Urinary albumin level of $\mathrm{Gas}^{-/-}$mice injected with Gas 6 and Gla $\Delta$ Gas 6 . Urinary albumin was collected 7 days after the injection of NTS. Each group consisted of seven mice, and data are expressed as mean \pm SD. Data from wild-type mice and $\mathrm{Gas}^{-1-}$ mice without treatment are consistent with the results shown in Figure 4b. (b) Number of PCNA-positive cells per glomerulus in $\mathrm{Gas}^{-/-}$mice injected with Gas 6 and Gla $\Delta$ Gas6. Mice were sacrificed on day 7 , and kidney sections were subjected to immunostaining with antiPCNA antibody. Each group contained eight mice, and 40 glomeruli per mouse were evaluated in a blinded fashion. Data are expressed as mean $\pm \mathrm{SD}$. Data from wild-type mice and $\mathrm{Gas}^{-/-}$mice without (c). Quantitative assessment of glomerular injury in $\mathrm{Gas}^{-/-}$mice injected with Gas6 and Gla $\Delta$ Gas6. Mice were sacrificed on day 21. Kidney sections were subjected to PAS staining. Data from wild-type mice and untreated $\mathrm{Gas}^{-/-}$mice are comparable to the results shown in Figure $5 \mathrm{~b}$. Each group contained seven mice, and 40 glomeruli per mouse were evaluated in a blinded fashion. Data are expressed as mean \pm SD. White bars, wild-type mice; black bars, $\mathrm{Gas}^{6^{-/}}$mice without treatment; light gray bars, Gas $6^{-/-}$mice injected with Gas6; dark gray bars, Gas6 $6^{-1-}$ injected with Gla $\Delta$ Gas6. ${ }^{*} P<0.05 ;{ }^{*} P<0.01 ;{ }^{*} P<0.001$.

Gas6 $6^{-1-}$ mice show similar deposition of sheep IgG and similar humoral immune response to sheep IgG. Humoral responses were examined as a potential explanation for the reduced disease severity in Gas $6^{-/-}$mice compared with wild-type mice. However, Gas $6^{-/}$mice had an immune response to sheep IgG similar to that of wildtype mice. Glomerular deposition of sheep IgG on day 7 was similar in both groups (Figure 7c). Serum titers of autologous antibody against sheep IgG were also comparable in the two groups on day 21 (Figure $7 \mathrm{~d}$ ).

Restoration of the Gas6-/- phenotype by recombinant Gas6, but not by Gla $\Delta$ Gas6. To confirm that reduced glomerular injury in $\mathrm{Gas}^{-/-}$mice was due to the deficiency of Gas6, we evaluated the effect of exogenously administered recombinant Gas6 (rGas6) on the development of NTN in $\mathrm{Gas}^{-/-}$mice. As a negative control, we used inactive Gas6 whose Gla domain was not $\gamma$-carboxylated (Gla $\Delta$ Gas6). Injection of rGas6 but not of Gla $\Delta$ Gas6 resulted in the death of three out of ten mice. Injection of rGas6 significantly increased proteinuria, while injection of Gla $\Delta$ Gas 6 did not (Figure 8a). We also examined the expression of PCNA on day 7 in Gas $6^{-/-}$mice injected with rGas6 or Gla $\Delta$ Gas6 (Figure 8b). Injection of Gas6 significantly increased the number of PCNA-positive cells per glomerulus, while injection of Gla $\Delta$ Gas 6 did not. We also evaluated the glomerular injury of Gas $6^{-/-}$mice injected with $\mathrm{rGas} 6$ and Gla $\Delta \mathrm{Gas} 6$ on day 21. Consistent with the increase in urinary protein, injection of rGas6 increased the percentage of sclerosing glomeruli, while injection of Gla $\Delta$ Gas 6 did not (Figure 8c). Injection of $\mathrm{rGas} 6$ induced more crescent formation than did Gla_Gas6, but the increase was not significant compared with uninjected $\mathrm{Gas}^{-/-}$mice. To confirm that injection of Gas 6 does not affect the renal morphology of mice, we injected the same amount of Gas6 into untreated wild-type mice $(n=6)$. We found no pathological change after injection of Gas6 in any mice (data not shown).

\section{Discussion}

In this study, we have shown that Gas6 was induced in NTN and that $\mathrm{Gas}^{-/}$mice were protected from glomerular injury by NTS. Gas $6^{-/-}$mice with NTN showed less mortality, less albuminuria, less glomerular injury, less proliferative response, and less deposition of fibrin/fibrinogen in glomeruli than wild-type mice did. Moreover, by the administration of rGas6, we could successfully induce severe glomerular injury in $\mathrm{Gas}^{-/-}$mice, indicating the essential role of Gas6 in this GN.

Accumulating data show that immune-mediated infiltration of leukocytes and monocytes is essential for the development of NTN $(9,17-21)$. However, in this report, we have shown that genetic deletion of Gas 6 inhibits the proliferative response, thereby improving the progression of nephritis in this model. These results suggest the possibility that proliferating glomerular cells may be therapeutic targets for inhibiting glomerular hypercellularity in lesions other than simple mesangioproliferative GN (8). In this regard, it was shown in rabbit NTN that proliferating marrow-derived monocytes/macrophages initially infiltrate the glomerulus and that epithelial cell proliferation subsequently contributes to the formation of crescents (22). Although additional studies will be necessary to determine whether glomerular epithelial cells are responsive to Gas6, recent studies by Shankland et al. have shown that glomerular epithelial cell proliferation is tightly regulated by cyclin-dependent kinase inhibitors and that this regulation can be released in crescentic GN (23).

Although we have tried to identify the origin of the PCNA-positive cells, most of the cells were negative for 
anti- $\alpha$ smooth muscle cell actin (data not shown). Furthermore, most of the cells were negative for immunostaining with anti-CD3, -CD45, and -CD68 (data not shown). We have not yet been able to determine the cell type of the PCNA-positive cells. This may be because there are no appropriate markers for mesangial or visceral epithelial cells in mice. Since we were concerned about the specificity of PCNA, we also determined the glomerular cell number in wild-type and $\mathrm{Gas}^{-/-}$mice and found a significant increase in cell number in wildtype mice. Although there is a possibility that some of the PCNA-positive cells are in an apoptotic stage, we assume that most of the PCNA-positive cells are proliferating. However, we do not definitely know that an increase in glomerular cell number is a prerequisite for the final glomerulosclerosis.

Part of the protective effect against NTN in $\mathrm{Gas}^{-/-}$ mice may be attributed to other biological functions of Gas6. Recently Angelillo-Scherrer et al. reported that deficiency of Gas 6 protects mice from thrombosis (24). In NTN, deposition of fibrin and fibrinogen is observed in glomeruli, and inhibition of thrombosis prevents severe renal damage $(25,26)$. We also confirmed less fibrin deposition in $\mathrm{Gas}^{-/-}$mice than in wild-type mice by electron microscopy (data not shown). Less deposition of fibrin and fibrinogen and renal injury in $\mathrm{Gas}^{-/-}$mice could be attributed to the thrombogenic effect of Gas6. Furthermore, Gas 6 can mediate chemotaxis of smooth muscle cells (27) and adhesion of Axl-expressing cells (28). Therefore, less proliferative response in glomerular cells in $\mathrm{Gas}^{-/-}$mice may be attributed to less migration of inflammatory cells into the glomerulus.

The cause of death in wild-type mice injected with NTS is not clear. Their lungs showed no evidence of hemorrhage. Because previous data reported rapid elevation of serum blood urea nitrogen during the first several days in this model (9) and bloody urine was noted in the bladders of dead animals, it is quite likely that the cause of death was renal failure.

As shown in Figure $6 \mathrm{c}$ and d, STAT3 was induced and phosphorylated in the proliferative phase, and its induction and phosphorylation was inhibited in $\mathrm{Gas}^{-/-}$mice. The molecular mechanism of STAT3 induction in NTN is unknown. Recent studies have shown transient induction of STAT3 in the nervous system after neuronal damage $(29,30)$ and in carotid artery remodeling after vascular injury (31). Therefore, it is possible that induction of STAT3 could be a ubi-quitous response to tissue injury in vivo. Furthermore, the composition of cells in the kidney is dramatically changed in the early phase of NTN and may account for the induction of STAT3 protein.

As for the experiments restoring the phenotype of Gas $6^{-/-}$mice, we injected the same dose of rGas6 to mice according to the dose used in the previous report. (24). In untreated Wistar rats, the serum concentration of Gas 6 is about $1-10 \mathrm{ng} / \mathrm{ml}$, and in Thy $1 \mathrm{GN}$, it increases in parallel with mesangial cell proliferation, reaching as high as $100 \mathrm{ng} / \mathrm{ml}$ (M. Yanagita, unpublished data). When $2 \mu \mathrm{g}$ of rGas6 is injected into $\mathrm{Gas}^{-/-}$mice weighing about
$20 \mathrm{~g}$, the serum concentration of rGas6 is expected to be $2 \mu \mathrm{g} / \mathrm{ml}$, which could be far more than that of endogenous Gas6 in wild-type mice. However, Axl on the surface of endothelial cells, monocytes, and macrophages $(32,33)$ could bind and eliminate rGas6 from the bloodstream. Furthermore, because this protein is obtained from rats, antibodies against rGas 6 could be produced and bind rGas6 before it reaches the site of inflammation. Therefore, sufficient amounts of rGas6 to reduce renal injury were injected daily into Gas $6^{-/-}$mice.

The question may arise whether renal injury in $\mathrm{Gas}^{-/-}$ mice injected with rGas6 can be attributed to the immunological reaction against rat protein. However, we thought it unlikely because urinary protein and PCNApositive cells per glomerulus in $\mathrm{Gas}^{-/-}$mice injected with rGas6 are increased as early as day 7 and because injection of the same amount of rat Gla $\Delta$ Gas 6 did not cause renal injury through immune reactions. Therefore, it is unlikely that an acute immunological reaction to heterologous rGas6 plays a role in this process.

However, in the later phase of NTN, the effect of immune reaction against rGas 6 cannot be ignored. Figure $8 \mathrm{c}$ shows that the injection of rGas 6 did not significantly stimulate the formation of crescents, despite the augmentation of glomerulosclerosis. Because the onset of crescent formation follows development of lesions in the glomerular capillaries, it is possible that elimination of rGas 6 by immunological response occurs before crescent formation. Alternatively, rGas6 may have greater access to cells within the glomerular capillaries than to those in the urinary space where crescents develop.

In conclusion, our data show that Gas6 is an essential factor in the development of NTN and suggest that strategies to eliminate Gas6 might protect against progressive renal injury and improve the prognosis of patients with kidney diseases. Further understanding of the Gas6 pathway may provide a therapeutic strategy in the treatment of progressive kidney diseases.

\section{Acknowledgments}

We thank Masashi Yanagisawa (University of Texas, Southwestern Medical Center), Toshiko Hori (Kyoto University) ,and Hideo Uchiyama(Taigenkai Hospital) for technical assistance. This study was supported by a Grant-in Aid from the Ministry of Education, Science, Sports, and Culture of Japan (13307034) and a Center of Excellence grant from the Japanese Ministry of Education, Science, Sports, and Culture (12CE2006). This work was also supported in part by United States Public Health Services research grant DK-30932 (to D.J. Salant). Motoko Yanagita is supported by the Japan Foundation for Aging and Health.

\footnotetext{
1. Floege, J., Topley, N., and Resch, K. 1991. Regulation of mesangial cell proliferation. Am. J. Kidney Dis. 17:673-676

2. Fogo, A., and Ichikawa, I. 1989. Evidence for the central role of glomerular growth promoters in the development of sclerosis. Semin. Nephrol. 9:329-342.

3. Striker, L.J., Doi, T., Elliot, S., and Striker, G.E. 1989. The contribution of glomerular mesangial cells to progressive glomerulosclerosis. Semin. Nephrol. 9:318-328.
} 
4. Manfioletti, G., Brancolini, C., Avanzi, G., and Schneider, C. 1993. The protein encoded by a growth arrest-specific gene (Gas6) is a new member of the vitamin $\mathrm{K}$-dependent proteins related to protein S, a negative coregulator in the blood coagulation cascade. Mol. Cell. Biol. 13:4976-4985.

5. Nakano, T., et al. 1995. Vascular smooth muscle cell-derived, Gla-containing growth-potentiating factor for $\mathrm{Ca}^{2+}$-mobilizing growth factors. J. Biol. Chem. 270:5702-5705.

6. Nakano, T., et al. 1997. Requirement of gamma-carboxyglutamic acid residues for the biological activity of Gas6: contribution of endogenous Gas 6 to the proliferation of vascular smooth muscle cells. Biochem. J. 323:387-392.

7. Yanagita, M., et al. 1999. Mechanism of inhibitory effect of warfarin on mesangial cell proliferation. J. Am. Soc. Nephrol. 10:2503-2509.

8. Yanagita, M., et al. 2001. Gas6 regulates mesangial cell proliferation through Axl in experimental glomerulonephritis. Am. J. Pathol. 158:1423-1432.

9. Topham, P.S., et al. 1999. Lack of chemokine receptor CCR1 enhances Th1 responses and glomerular injury during nephrotoxic nephritis. J. Clin. Invest. 104:1549-1557.

10. Chugh, S., et al. 2001. Aminopeptidase A: a nephritogenic target of nephrotoxic serum. Kidney Int. 59:601-613.

11. Thomas, K.R., and Capecchi, M.R. 1987. Site-directed mutagenesis by gene targeting in mouse embryo-derived stem cells. Cell. 51:503-512.

12. Ledermann, B., and Burki, K. 1991. Establishment of a germ-line competent C57BL/6 embryonic stem cell line. Exp. Cell Res. 197:254-258.

13. Suzuki, Y., et al. 1998. Distinct contribution of $\mathrm{Fc}$ receptors and angiotensin II-dependent pathways in anti-GBM glomerulonephritis. Kidney Int. 54:1166-1174

14. Bellosta, P., Zhang, Q., Goff, S.P., and Basilico, C. 1997. Signaling through the ARK tyrosine kinase receptor protects from apoptosis in the absence of growth stimulation. Oncogene. 15:2387-2397.

15. Goruppi, S., Ruaro, E., Varnum, B., and Schneider, C. 1997. Requirement of phosphatidylinositol 3-kinase-dependent pathway and Src for Gas6Axl mitogenic and survival activities in NIH 3 T3 fibroblasts. Mol. Cell. Biol. 17:4442-4453.

16. Yanagita, M., et al. 2001. Gas6 induces mesangial cell proliferation via latent transcription factor STAT3. J. Biol. Chem. 276:42364-42369.

17. Hebert, M.J., et al. 1998. Acute nephrotoxic serum nephritis in complement knockout mice: relative roles of the classical and alternate pathways in neutrophil recruitment and proteinuria. Nephrol. Dial. Transplant. 13:2799-2803

18. Park, S.Y., et al. 1998. Resistance of $F_{C}$ receptor-deficient mice to fatal glomerulonephritis. J. Clin. Invest. 102:1229-1238.

19. Rosenkranz, A.R., Mendrick, D.L., Cotran, R.S., and Mayadas, T.N. 1999.
P-selectin deficiency exacerbates experimental glomerulonephritis: a protective role for endothelial P-selectin in inflammation. J. Clin. Invest. 103:649-659.

20. De Vriese, A.S., et al. 1999. The role of selectins in glomerular leukocyte recruitment in rat anti-glomerular basement membrane glomerulonephritis. J. Am. Soc. Nephrol. 10:2510-2517.

21. Yokoo, T., et al. 2001. Genetically modified bone marrow continuously supplies anti-inflammatory cells and suppresses renal injury in mouse Goodpasture syndrome. Blood. 98:57-64.

22. Cattell, V., and Jamieson, S.W. 1978. The origin of glomerular crescents in experimental nephrotoxic serum nephritis in the rabbit. Lab. Invest. 39:584-590.

23. Shankland, S.J., et al. 2000. Differential expression of cyclin-dependent kinase inhibitors in human glomerular disease: role in podocyte proliferation and maturation. Kidney Int. 58:674-683.

24. Angelillo-Scherrer, A., et al. 2001. Deficiency or inhibition of Gas6 causes platelet dysfunction and protects mice against thrombosis. Nat. Med. 7:215-221.

25. Nakamoto, Y., et al. 1978. Microangiographic evaluation of the effects of heparin on progressive Masugi nephritis. Kidney Int. 13:297-305.

26. Wu, X., Helfrich, M.H., Horton, M.A., Feigen, L.P., and Lefkowith, J.B. 1994. Fibrinogen mediates platelet-polymorphonuclear leukocyte cooperation during immune-complex glomerulonephritis in rats. J. Clin. Invest. 94:928-936.

27. Fridell, Y.W., Villa, J., Jr., Attar, E.C., and Liu, E.T. 1998. GAS6 induces Axl-mediated chemotaxis of vascular smooth muscle cells. J. Biol. Chem. 273:7123-7126.

28. McCloskey, P., et al. 1997. GAS6 mediates adhesion of cells expressing the receptor tyrosine kinase Axl. J. Biol. Chem. 272:23285-23291.

29. Acarin, L., Gonzalez, B., and Castellano, B. 1998. Stat3 and NFkappaB glial expression after excitotoxic damage to the postnatal brain. Neuroreport. 9:2869-2873.

30. Acarin, L., Gonzalez, B., and Castellano, B. 2000. STAT3 and NFkappaB activation precedes glial reactivity in the excitotoxically injured young cortex but not in the corresponding distal thalamic nuclei. J. Neuropathol. Exp. Neurol. 59:151-163.

31. Seki, Y., et al. 2000. Role of the JAK/STAT pathway in rat carotid artery remodeling after vascular injury. Circ. Res. 87:12-18.

32. Avanzi, G.C., et al. 1998. GAS6 inhibits granulocyte adhesion to endothelial cells. Blood. 91:2334-2340.

33. Neubauer, A., et al. 1994. Expression of Axl, a transforming receptor tyrosine kinase, in normal and malignant hematopoiesis. Blood. 84:1931-1941. 